$\mathrm{SCIDOC}$

International Journal of Reproduction, Fertility \& Sexual Health (IJRFSH)

ISSN: $2377-1887$

\title{
Emergency And Elective Gynecological Surgery Morbidityin Covid 19 Pandemic
}

\section{Research Article}

Melike Doganay, Rahime Bedir Findik*, Kuntay Kokonali, Orhan Seyfi Aksakal, Cemal Reşat Atalay, Kadir Çetinkaya

Department of Obstetrics and Gynecology, Ankara City Hospital Medical Hospital 5 (MH5) Ankara, Turkey.

Abstract

Objectives: In this study, we aimed to investigate the surgical morbidity changes in the surgical team behavior created by the pandemic in emergency and elective gynecological operations.

Materials and Methods: A total of 100 patient files undergoing 50 emergency and 50 elective gynecological operations with benign indications between March 10 and May 30, 2020 were retrospectively reviewed.After recording the demographic characteristics of the two groups, the two groups were compared for intraoperative and postoperative complications and morbidity. Statistical analyses were performed using Statistical Package for the Social Sciences version 17.0 (SPSS Inc, Chicago, IL). groups were compared using the Kolmogorov-Smirnov test, Student's t test and Chi-square test.

Results: The average age of the emergency cases was statistically significantly lower than the electives $(p<0.001)$. Pregnancy and delivery numbers of the patients were also statistically significantly lower in the emergency group $(p=0.028)$. In emergency cases, previous pelvic surgery and the presence of adhesions due to this were statistically significantly higher $(\mathrm{p}=0.050$; $\mathrm{p}=0.021)$. Operation time and presence of systemic disease were significantly higher in the elective group $(\mathrm{p}=0.001 ; \mathrm{p}=0.046)$. It was determined that endoscopic surgery was preferred significantly more than elective in emergency cases $(\mathrm{p}=<0.001)$. It was determined that presence of obesity (Odds ratio $=1.54, \% 95 \mathrm{CI}=0.61-3.87, \mathrm{p}=0.019)$ and adhesion (Odds ratio $=4.21$; $\% 95 \mathrm{CI}=1.26-14.04 ; \mathrm{p}<0.001)$ were the independent factors related to surgical morbidity.

Conclusions: Laparoscopic procedures seem to be safer because they reduce postoperative morbidities in patients who are hemodynamically stable and have a very good preoperative evaluation in the Covid 19 pandemic.

Keywords: Gynecologic Surgery; Laparoscopy; Laparotomy; Surgical Morbidity; Covid 19 Pandemic.

\section{Introduction}

The new type of coronavirus disease was detected on January 13, 2020 and was described by the World Health Organization as a pandemic that threatens the health of the whole world on March 11, 2020 [1]. It spread rapidly, killing about 3 million people. This number is expected to increase gradually.

The pandemic has significantly consumed existing health resources and a significant portion of the human resources allocated for health have been directed to this area. Of course, in this case, the operation of the surgical branches had to be changed. Government policies have been developed throughout the country, taking into account the recommendations of the relevant surgical committees in order not to interrupt the health service provided.
According to the decisions taken regarding benign gynecological operations in our country, non-urgent operations were suspended for 3 months at the beginning of the pandemic and emergency cases were prioritized as much as possible.

Pandemic may have made a change in the behavior of surgeons, as in all people, due to the fact that covid 19 is a contagious and lethal disease, progresses with different clinical symptoms from person to person, does not have a very effective vaccine yet and is a very newly described disease. For example, the surgeon may want to complete the surgery he has entered in a shorter time. Completing the operation as soon as possible, although protective measures such as a mask are taken, may be important for the patient and the entire team, including the patient, in terms of minimizing the risk of contamination, as it will take anesthesia for

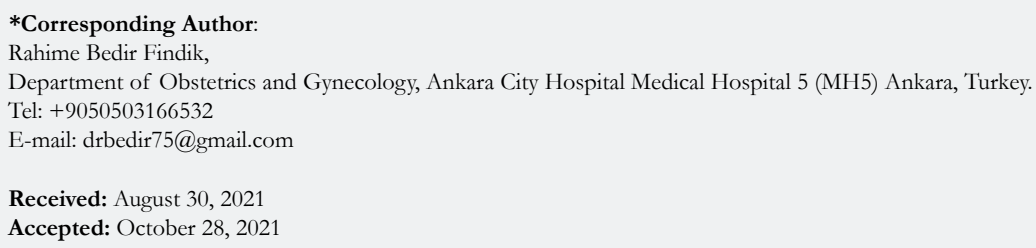

Copyright: Rahime Bedir Findik ${ }^{\circ}$ 2021. This is an open-access article distributed under the terms of the Creative Commons Attribution License, which permits unrestricted use, distribution and reproduction in any medium, provided the original author and source are credited. 
a shorter time.

In this study, as Ankara City Hospital Gynecology Clinic, we aimed to investigate the surgical morbidity changes in the surgical team behavior created by the pandemic in emergency and elective gynecological operations.

\section{Materials And Method}

After obtaining approval from the Ankara City Hospital Ethics Committee and Ministry of Health Scientific Research Platform, a total of 100 patient files undergoing 50 emergency and 50 elective gynecological operations with benign indications between March 10 and May 30, 2020 were retrospectively reviewed. Our work complies with the provisions of the helsingi declaration.

Demographic characteristics (age, number of pregnancy, body mass index, presence of cesarean section in the anamnesis, previous pelvic surgery, presence of systemic disease), laboratory findings (pre- and postoperative hemoglobin values), operative properties (type of surgery, presence of adhesion, operation time, mean amount of bleeding during the operation), intra- or postoperative complications and duration of postoperative hospitalization of all patients were recorded.
Presence of any conditions including blood transfusion, adjacent organ injury, prolonged operation time (>180 min), reoperation, prolonged ( $>5$ days) hospitalization or detection of wound infection was classified as surgical morbidity.

Statistical analyses were performed using Statistical Package for the Social Sciences version 17.0 (SPSS Inc, Chicago, IL). Normal distribution of data was assessed using Kolmogorov-Smirnov test. Continuous and normally distributed variables were presented as mean \pm standart deviation, and intragroup differences were evaluated using Student's t test. Categorical variables were expressed as number (percentage). Differences between categorical data were analyzed using Chi-square test. Odds ratios and 95\% confidence intervals (CIs) of independent factors for surgical morbidity were calculated via multiple logistic regression model. A p value of less than 0.05 was taken to be significant.

\section{Results}

The characteristics of elective and emergency patients are presented in Table 1. Accordingly, the average age of the emergency cases was statistically significantly lower than the electives. Pregnancy and delivery numbers of the patients were also statistically

Table 1. Characteristics of the groups.

\begin{tabular}{|c|c|c|c|}
\hline & Elective & Emergency & \multirow[t]{2}{*}{$\mathbf{P}$} \\
\hline & $(n=50)$ & $(n=50)$ & \\
\hline Age (years) & $47.04 \pm 11.10$ & $36.88 \pm 10.77$ & $<0.001$ \\
\hline Gravidy & $3.34 \pm 1.98$ & $2.56 \pm 1.49$ & 0.028 \\
\hline Parity & $2.80 \pm 1.67$ & $1.98 \pm 1.30$ & 0.008 \\
\hline BMI $(\mathrm{kg} / \mathrm{m} 2)$ & $29.05 \pm 4.64$ & $27.06 \pm 7.74$ & 0.121 \\
\hline Preop $\mathrm{Hg}(\mathrm{gr} / \mathrm{dl})$ & $12.25 \pm 1.80$ & $12.01 \pm 1.61$ & 0.487 \\
\hline Postop Hg (gr/dl) & $11.40 \pm 1.60$ & $11.29 \pm 1.28$ & 0.281 \\
\hline $\mathrm{Hg}$ exchange $(\mathrm{gr} / \mathrm{dl})$ & $0.84 \pm 0.28$ & $0.92 \pm 0.36$ & 0.739 \\
\hline Amount of estimated bleeding (cc) & $171.80 \pm 50.79$ & $181.20 \pm 75.86$ & 0.775 \\
\hline Previous cesarean section & $11(22.0)$ & $18(36.0)$ & 0.123 \\
\hline Presence of systemic disease & $14(28.0)$ & $6(12.0)$ & 0.046 \\
\hline Previous pelvic surgery & $4(8.0)$ & $11(22.0)$ & 0.05 \\
\hline \multicolumn{3}{|l|}{ Type of surgery performed } & \multirow[t]{5}{*}{$<0.001$} \\
\hline Laparoscopy & $1(2.0)$ & $13(26.0)$ & \\
\hline Hysteroscopy & $4(8.0)$ & $1(2.0)$ & \\
\hline Abdominal (Laparotomy) & $30(60.0)$ & $35(70.0)$ & \\
\hline Vaginal & $15(30.0)$ & $1(2.0)$ & \\
\hline Presence of adhesion & $3(6.0)$ & $11(22.0)$ & 0.021 \\
\hline \multicolumn{3}{|l|}{ Adjacent organ injury } & \multirow[t]{3}{*}{0.36} \\
\hline Bladder & $2(4.0)$ & $0(0.0)$ & \\
\hline Bovel & $1(2.0)$ & $1(2.0)$ & \\
\hline Reoperation & $1(2.0)$ & $0(0.0)$ & 0.5 \\
\hline Operation time (second) & $117.30 \pm 52.41$ & $84.44 \pm 38.41$ & 0.001 \\
\hline Time of hospitalization (day) & $2.63 \pm 0.98$ & $2.76 \pm 0.53$ & 0.715 \\
\hline Wound infection & $0(0.0)$ & $1(2.0)$ & 0.5 \\
\hline \multicolumn{4}{|c|}{ Values were given as mean \pm standard deviation, number (percentage). } \\
\hline \multicolumn{4}{|c|}{$\mathrm{p}<0.05$ was considered statistically significant } \\
\hline
\end{tabular}


significantly lower in the emergency group. In emergency cases, previous pelvic surgery and the presence of adhesions due to this were statistically significantly higher. Operation time and presence of systemic disease were significantly higher in the elective group.

Of the 50 patients who were operated electively, $13(26 \%)$ underwent prolapse surgery due to prolapse or incontinence; 24 $(48 \%)$ had myoma due to bleeding and underwent myomectomy or hysterectomy; 4 (8\%) underwent operative hysteroscopy due to endometrial polyp; 7 (14\%) underwent cystectomy due to benign cysts of the ovary; $1(2 \%)$ consisted of patients who underwent bartholin cystectomy and $1(2 \%)$ laparoscopic bilateral tubal ligation. $17(34 \%)$ of 50 emergency patients were admitted due to ectopic pregnancy; 12 (24\%) had cyst operation; 11 (22\%) had acute bleeding due to myoma, $8(16 \%)$ were ovarian or adnexal torsion; 2 (4\%) were operated for tubooverian ruptured and painful abscess.

Surgical morbidity was detected in $19(19 \%)$ patients. The presence of obesity $(57.9 \%$ vs $15.9 \%)$, open abdominal surgery $(94.7 \%$ vs $58.0 \%)$ and adhesion $(47.2 \%$ vs $6.2 \%)$ was higher in these patients than in the no surgical morbidity group. But, the presence of elective or emergency operation was similar in groups with and without surgical morbidity. (Table 2).

According to the results of multiple regression analysis shown in Table 3, it was determined that presence of obesity (Odds ratio $=1.54, \% 95 \mathrm{CI}=0.61-3.87, \mathrm{p}=0.019)$ and adhesion (Odds ratio $=4.21 ; \% 95 \mathrm{CI}=1.26-14.04 ; \mathrm{p}<0.001)$ were the independent factors related to surgical morbidity.

\section{Discussion}

With the spread of the virus all over the world and in our country, it has become necessary to make serious changes in the health sector. The main purpose here is to prevent the accumulation, burdening and locking in the health system and 3rd level intensive care units with the first wave of virus infection. Therefore, elective outpatient services and postponement of all elective surgical cases have come to the fore. International and national gynecology and obstetrics associations have published their opinions on this issue $[2,3]$. According to these guidelines, it is recommended that all elective gynecological surgeries be postponed in countries where COVID-19 virus infection is seen, including our country.

Table 2. Comparison of the groups according to the presence of surgical morbidity.

\begin{tabular}{|c|c|c|c|}
\hline & No surgical morbidity & Surgical morbidity & \multirow{2}{*}{$\mathbf{P}$} \\
\hline & $(n=81)$ & $(n=19)$ & \\
\hline Age & $41.19 \pm 12.42$ & $45.26 \pm 9.72$ & 0.185 \\
\hline Gravidy & $2.84 \pm 1.79$ & $3.42 \pm 1.74$ & 0.202 \\
\hline Parity & $2.32 \pm 1.56$ & $2.68 \pm 1.49$ & 0.361 \\
\hline BMI & $27.72 \pm 6.81$ & $29.51 \pm 4.31$ & 0.277 \\
\hline Obesity (BMI $\geq 30 \mathrm{~kg} / \mathrm{m} 2)$ & $21(15.9)$ & $11(57.9)$ & 0.007 \\
\hline \multicolumn{3}{|l|}{ Groups } & \multirow{3}{*}{0.202} \\
\hline Elective surgery & $38(46.9)$ & $12(63.2)$ & \\
\hline Emergency surgery & $43(53.1)$ & $7(36.8)$ & \\
\hline Prvious cesarean section & $20(24.7)$ & $9(47.4)$ & 0.059 \\
\hline Presence of systemic disease & $18(22.2)$ & $2(10.5)$ & 0.251 \\
\hline Previous pelvic surgery & $11(13.6)$ & $4(21.1)$ & 0.412 \\
\hline \multicolumn{3}{|l|}{ Type of surgery performed } & \multirow{5}{*}{0.025} \\
\hline Laparoscopy & $14(17.3)$ & $0(0.0)$ & \\
\hline Hysteroscopy & $5(6.2)$ & $0(0.0)$ & \\
\hline Abdominal (Laparotomy) & $47(58.0)$ & $18(94.7)$ & \\
\hline Vaginal & $15(18.5)$ & $1(5.3)$ & \\
\hline Presence of adhesion & $5(6.2)$ & $9(47.4)$ & $<0.001$ \\
\hline \multicolumn{4}{|c|}{ Values were given as mean \pm standard deviation, number (percentage). } \\
\hline \multicolumn{4}{|c|}{$\mathrm{p}<0.05$ was considered statistically significant } \\
\hline
\end{tabular}

Table 3. Multiple regression analysis results of risk factors for surgical morbidity.

\begin{tabular}{|c|c|c|c|c|}
\hline & Wald & SE & p & OR (\%95 CI) \\
\hline Type of surgery performed & 0.85 & 0.47 & 0.357 & $0.07(0.02-0.27)$ \\
\hline Presence of obesity & 5.46 & 0.615 & 0.019 & $1.54(0.61-3.87)$ \\
\hline Presence of adhesion & 14.61 & 0.701 & $<0.001$ & $4.21(1.26-14.04)$ \\
\hline SE: Standard error; OR: Odds ratio; CI: Confidence interval \\
\hline p <0.05 was considered statistically significant \\
\hline
\end{tabular}


In cases where delay and delay do not threaten the life of the patient, planning the surgery for a later date will keep the patient at home, prevent possible transmission and prevent possible burden on the health system. Conditions that endanger the life of the patient according to the guideline published by the American College of Surgeons on March 24, 2020; ectopic pregnancy, adnexal torsion, ruptured tubal-ovarian abscess, tubo-ovarian abscess not responding to conservative treatment, acute, severe vaginal bleeding and emergency cerclage It was defined as [3]. In these cases, surgery should be performed without delay, taking the necessary precautions.

In this study, we compared the intraoperative complications and postoperative morbidity rates of cases that were taken urgently and electively during the Covid 19 pandemic. The Covid 19 pandemic caused us to delay elective cases as much as possible. It was important that surgeons, whether elective or emergency, tried to complete the case as soon as possible, in terms of not putting themselves and the surgical team at risk.

None of our emergency or elective cases had complications related to covid-19. PCR tests of all patients were negative. The average age of our patients who underwent emergency gynecological surgery was significantly lower than that of patients who were taken electively. The reason for this is that 34\% of our emergency cases were operated for ectopic pregnancy and $24 \%$ of them were operated due to ovarian cysts, that is, more than half of the emergency patients are common diseases in the reproductive age. The remaining half were due to acute bleeding myoma, torsion and abscess.

Since those who underwent emergency surgery were in the younger age group, the total number of pregnancies and the number of births were also lower than the elective group. Remarkably, patients who underwent emergency surgery had previously undergone pelvic surgery compared to electives, which explains why ectopic pregnancy is the first in this group. The fact that $26 \%$ of elective operations were performed vaginally due to prolapse and incontinence and $48 \%$ consisted of patients operated abdominally due to bleeding or symptomatic fibroids.

Publications about performing the surgery with laparotomy and laparoscopy do not necessarily indicate laparotomy. The main concern for minimally invasive surgery is patient groups that are asymptomatic and not known to be infected. In such patients, there is a risk of contamination from viral particles during the intubation and extubation process and during the formation of pneumoperitoneum. In addition, there is a study in a study that asymptomatic patients increase the need for postoperative intensive care and mortality after surgery [4].

In the Covid 19 pandemic, laparotomy operations were preferred instead of laparoscopic operations. Laparoscopy may be prominent only in the emergency patient group, since patients are operated mostly due to ectopic reasons. In addition, we decided to perform laparoscopy on the patient by making a very good preoperative evaluation. The rate of adhesion due to pelvic surgery was higher in the emergency group than elective, and this result indicated the presence of adhesion secondary to previous pelvic surgery.

When the operation times of both groups were compared, the duration of the emergency patient group was naturally determined less than the other.

In our study, re-operation of the patient, need for blood transfusion, adjacent organ injury, wound site infection, hospital stay of more than 5 days were evaluated as indicators of morbidity. When these parameters are evaluated, although there is no difference between emergency and elective morbidities, the presence of adhesion and obesity appear to be the two factors that affect morbidity the most. When we paid attention to the type of surgery performed, it was found that $94.7 \%$ of the cases with morbidity were in those with laparotomy and $5.3 \%$ in those who had vaginal surgery. In this respect, although endoscopic operations such as laparoscopic and hysteroscopy are a more reliable type of surgery than others, it has ceased to be a preferred type of surgery on the grounds that asymptomatic and infected patients pose a risk during the Covid Period. However, it still maintains its importance as the preferred surgical technique in very well selected patient groups.

In previous review and meta-analysis studies related to laparoscopic and laparotomy, it has been argued that laparoscopy is a safer procedure and reduces postoperative complications when applied to hemodynamically stable patients compared to laparotomy [5].

In terms of the type of surgery, it is thought that viral particles that may be in the tissue and blood in the laparoscopic approach may occur during the pneumoperitoneum formation phase, the gas escaping while inserting and removing instruments from the trocars, and during coagulation, but so far, no disadvantage of minimai invasive surgery to laparotomy has been scientifically reported. Nevertheless, the theoretical risk was minimized by working at low pressures $(8-10 \mathrm{~mm} \mathrm{Hg})$, especially during laparoscopic surgery, and avoiding tissue coagulation as much as possible by preventing the leakage of $\mathrm{CO}_{2}$ gas into the room air with aspiration cannulae [6].

However, in the Covid 19 Pandemic, the patients should be evaluated very well pre-intra and postop for laparoscopy and laparotomy decision. In the preoperative period, the contact history of the patient should be evaluated. Traveling to a foreign country or center where prevalence is high, contact with people coming from this locality should be questioned. The patient should be asked about symptoms such as the presence of URI symptoms (cough, fever, headache, mylaji). In non-urgent cases, PCR test is also required preoperatively. and in this way, laparoscopic surgery can be performed safely [7].

In emergency cases or in situations where pre-op covid testing is not possible, it is important to consider every patient risky in terms of covid. Personal protective equipment (PPE; apron, N-95/FFP2-3 mask, visor, pair of gloves) must be worn in patients known to have Covid. In other cases, it is stated that the surgical mask will be sufficient in terms of protection. None of our patients were covid-positive.

\section{Conclusion}

In conclusion laparoscopic procedures seem to be safer because they reduce postoperative morbidities in patients who are hemo- 
dynamically stable and have a very good preoperative evaluation in the Covid 19 pandemic. We need to be more careful in abdominal operations and laparoscopic operations, especially in selected patients who are obese and have adhesions.

\section{References}

[1]. WHO Timeline - COVID-19 2020. Available from: https://www.who.int/ news-room/detail/27-04-2020-who-timeline---covid-19.

[2]. http://www.mijid.org/upload/2020/04/mijid-covid19-13 April.

[3]. American College of Surgeons Facts Sheet: Create a Surgical Review Committee for COVID-19-Related Surgical Triage Decision Making
[4]. Aminian A, Safari S, Razeghian-Jahromi A, Ghorbani M, Delaney CP. COVID-19 Outbreak and Surgical Practice: Unexpected Fatality in Perioperative Period. Ann Surg. 2020 Jul; 272(1): e27-e29. PMID: 32221117.

[5]. Hajibandeh S, Hajibandeh S, Gumber AO, Wong CS. Laparoscopy versus laparotomy for the management of penetrating abdominal trauma: a systematic review and meta-analysis. International Journal of Surgery. 2016 Oct 1; 34: 127-36.

[6]. Mallick R, Odejinmi F, Clark TJ. Covid 19 pandemic and gynaecological laparoscopic surgery: knowns and unknowns. Facts Views Vis Obgyn. 2020 Apr 1; 12(1): 3-7. PMID: 32259155.

[7]. AAGL - Elevating Gynecologic Surgery. Joint Society Statement on Elective Surgery during COVID-19 Pandemic. J Minim Invasive Gynecol. 2020 JulAug;27(5):1033. PMID: 32512207. 